TRABAJOS ORIGINALES

Rev Obstet Ginecol Venez. 2021; 81 (2): 108-115

https://doi.org/10.51288/00810204

\title{
Aspiración manual endouterina en comparación con el legrado uterino instrumental en el tratamiento del aborto incompleto o retenido*
}

\author{
Yetzabeth del Valle Mencia Flores, ${ }^{1}$ (i) Maria Alejandra Santiago Villegas, ${ }^{1}$ Jesús Fernández Pinto. ${ }^{2}$
}

\begin{abstract}
RESUMEN
Objetivo: Comparar la eficacia de la aspiración manual endouterina con el legrado uterino instrumental como tratamiento del aborto incompleto o retenido en pacientes que consultan a la Maternidad Concepción Palacios entre julio y diciembre 2015.

Métodos: Previo consentimiento informado, se distribuyeron al azar 192 pacientes en dos grupos, a un grupo se le practicó aspiración manual endouterina con anestesia paracervical y al otro, legrado uterino instrumental con sedación endovenosa. Se estudió la capacidad para evacuar los restos, la tolerancia y la duración de los procedimientos.

Resultados: La cantidad de restos ovulares medidos por ecografía transvaginal después del vaciamiento fue entre $3 \mathrm{~mm}$ y $4 \mathrm{~mm}$ en 73 pacientes (76,0\%) a quienes se les practicó legrado y en 71 (74,0\%) del grupo aspiración $(p=0,2145)$. El dolor durante la aspiración fue leve en 93 pacientes $(96,9 \%)$. A los 10 minutos del procedimiento, todas las pacientes de ambos grupos refirieron entre 0 y 3 puntos en la escala del dolor. En el grupo de aspiración no se presentaron efectos colaterales relacionados con la anestesia, en el grupo legrado se observó náuseas en 2 casos (2\%) y mareos en 1 (1\%). No se presentaron complicaciones inherentes al procedimiento (retención de restos, perforaciones ni infección) en ninguno de los dos grupos. La intervención tardó 8,45 $\pm 1,42$ minutos y 8,50 $\pm 1,32$ minutos, en los grupos legrado y aspiración, respectivamente $(p=0,8138)$. Conclusiones: La aspiración y el legrado son eficaces y bien tolerados en el tratamiento del aborto.
\end{abstract}

Palabras clave: Legrado Uterino, Aborto Incompleto, Aborto Retenido, Aspiración Manual Endouterina.

\section{SUMMARY}

Objective: To compare the effectiveness of manual endouterine aspiration with instrumental uterine curettage as a treatment for incomplete or retained abortion in patients who consult to the Hospital "Maternidad Concepción Palacios between July and December 2015.

Methods: Upon informed consent, the 192 patients were randomly distributed in two groups; one group was given manual endouterine aspiration with paracervical anesthesia and to the other, instrumental uterine curettage with endovenous sedation. The ability to evacuate the remains, tolerance and duration of procedures was studied.

Results: The amount of ovular remains measured by transvaginal ultrasound after emptying was between $3 \mathrm{~mm}$ and $4 \mathrm{~mm}$ in 73 patients (76.0\%) who underwent curettage and in $71(74.0 \%)$ of the aspiration group $(p=0.2145)$. Pain during aspiration was mild in 93 patients (96.9\%). At 10 minutes of the procedure, all patients in both groups reported between 0 and 3 points on the pain scale. In the aspiration group there were no side effects related to anesthesia, in the curettage group nausea was observed in 2 cases (2\%) and dizziness in 1 (1\%). There were no complications inherent in the procedure (retention of remains, perforations or infection) in either group. The intervention took $8.45 \pm 1.42$ minutes and $8.50 \pm 1.32$ minutes in the curettage and aspiration groups, respectively $(p=0.8138)$.

Conclusions: Aspiration and curettage are effective and well tolerated in the management of abortion.

Keywords: Uterine curettage, Incomplete Abortion, Abortion retained, Vacuum aspiration.

${ }^{1}$ Obstetras ginecólogos, egresados de la Universidad Central de Venezuela, sede Maternidad Concepción Palacios. ${ }^{2}$ Jefe de Servicio de Sala de Partos de la Maternidad Concepción Palacios.

* Trabajo Especial de Grado presentado y aprobado en la Universidad Central de Venezuela para optar al título de Especialista en Obstetricia y Ginecología.

\section{INTRODUCCIÓN}

El aborto se considera la complicación más frecuente de la gestación, sin embargo resulta difícil precisar su 
frecuencia. Se ha estimado una incidencia de $15 \%$ a $20 \%$ de todas las gestaciones (1).

Cada año, se calcula que quedan embarazadas 208 millones de mujeres. Entre ellas, el 59 \% tiene un embarazo planificado o deseado que culmina con un nacimiento con vida, un aborto espontáneo o una muerte fetal intrauterina. El $41 \%$ restante de los embarazos no es deseado (2). Según la Organización Mundial de la Salud (OMS), se calcula que cada año se realizan 22 millones de abortos peligrosos en todo el mundo, casi todos ellos en países en desarrollo; lo que produce la muerte de alrededor de 47000 mujeres, lo que representa el $13 \%$ de todas las defunciones maternas a nivel mundial y discapacidades en otros 5 millones de mujeres (2-4). En América Latina y El Caribe, se llevan a cabo aproximadamente 4 millones de abortos en condiciones de riesgo al año y, específicamente, América del Sur muestra la razón de abortos inseguros más alta del mundo, 38 abortos por cada 100 nacidos vivos (5).

Una vez confirmado el diagnóstico de aborto y la necesidad de realizar la evacuación uterina, se cuenta con diferentes alternativas para lograr esta meta: el manejo expectante, el manejo médico y el manejo quirúrgico, que incluye el legrado uterino instrumental (LUI) y la aspiración manual endouterina (AMEU) de más reciente uso, pero con ventajas que superan el método tradicional. Debe seleccionarse adecuadamente el manejo según las características de la paciente, la edad gestacional, las condiciones clínicas y los recursos de cada institución.

Dentro de los procedimientos quirúrgicos, la OMS recomienda el uso de legrado uterino por aspiración para el tratamiento del aborto, en virtud de presentar mayores ventajas en comparación con el método tradicional de legrado uterino instrumental con cureta; la evidencia ha demostrado que se requiere de menor dilatación cervical, menos uso de anestésicos, menor sangrado y menor tiempo de estancia hospitalaria, lo que resulta en menores costos para la salud pública (4, 6-8). Sin embargo, en algunas instituciones nacionales ha habido ciertas dificultades para la introducción de la AMEU y se observa que aún se utiliza el LUI.

Siendo el aborto una causa importante de morbimortalidad a nivel mundial, es imperativa la aplicación de medidas eficaces y seguras para el tratamiento del mismo y para contribuir a la disminución de dichas tasas. El aborto incompleto es un problema importante que debe tratarse con efectividad mediante procedimientos seguros y adecuados.

Debido a la alta prevalencia de abortos a nivel mundial y, especialmente, en Latinoamérica y El Caribe, surge la inquietud de realizar este trabajo de investigación y así permitir una mejor calidad de atención para las pacientes con disminución del riesgo de las complicaciones inherentes al procedimiento quirúrgico utilizado.

Se han realizado múltiples investigaciones en las que se evalúa la AMEU como técnica de tratamiento del aborto, todos con resultados similares. En 2007, Forna y cols. (9), publicaron una revisión de Cochrane sobre procedimientos quirúrgicos para la evacuación del aborto espontáneo incompleto, en la cual comparaban la seguridad y efectividad de los métodos de evacuación uterina, desde 1966 hasta diciembre de 2002, obtuvieron como resultados que la vacuoextracción se asoció con una pérdida de sangre significativamente menor desde el punto de vista estadístico, menos dolor y una menor duración del procedimiento con relación al legrado con cureta. Las complicaciones graves, como la perforación uterina $\mathrm{y}$ otros tipos de morbilidad, fueron poco frecuentes. Resultados similares han reportado otros autores (6, 10, 11). En 2009, Kulier y cols. (12) publicaron una nueva revisión Cochrane sobre el tema y reportaron que el uso de la AMEU no se asoció a diferencias estadísticamente significativas para la pérdida excesiva de sangre, transfusión de sangre, morbilidad febril, 
retención de restos posevacuación, reingresos, dolor o el uso de antibióticos terapéuticos; sin embargo si hubo diferencia estadísticamente significativa en la duración del procedimiento, siendo más corto en la aspiración endouterina que en el legrado uterino instrumental. Además de las características descritas, se han encontrado como ventajas, una menor pérdida sanguínea, menos tiempo en el procedimiento, menor estancia hospitalaria y por lo tanto menor costo; el procedimiento no requiere anestesia general y la tasa de complicaciones es menor que la dilatación y curetaje. Estos resultados han sido avalados por otros estudios que señalan que el uso de AMEU en el tratamiento del aborto en el primer trimestre es práctico, seguro, barato y con ahorro de tiempo, estrategia útil para reducir la mortalidad y la morbilidad (13-17). Finalmente, también se ha reportado una reducción en el desarrollo de síndrome de Asherman en las pacientes a quienes se les realizó AMEU o a quienes recibieron tratamiento médico, al compararlas con aquellas a quienes se les había realizado el legrado uterino instrumental (18).

El manejo del aborto incluye tratamiento médico o quirúrgico. Históricamente los primeros legrados uterinos instrumentales datan del año 1846, cuando el cirujano y ginecólogo francés Joseph Claude Anthélme Recamier del Hôtel Dieu de Paris, introdujo la cureta con la forma que se conoce actualmente para realizar curetaje uterino; en 1886 se incorporaron los dilatadores del cuello, elaborados en metal. Durante el siglo XX, en el año 1924, Howard Atwood Kelly inició la era de legrados en el consultorio, con sedación o sin ningún tipo de anestesia. Emil Novak diseñó su cánula o cureta de pequeño calibre y punta aserrada para hacer biopsias en 1935. En ese mismo año, Novak diseñó un sistema de aspiración y legrado mediante la utilización de un vacuum conectado a tubos aserrados (de mayor calibre que la cureta de Novak). De esta manera se inició la modalidad de aspiración y legrado de la cavidad endometrial. En 1982, Cornier diseñó una cureta flexible para conectar a un aspirador llamado Vabra. A inicios de los 90, fue la introducción al arsenal quirúrgico del equipo de Karman y la técnica de aspiración manual endouterina, que hoy en día es recomendada por la OMS y la FIGO para el manejo seguro del aborto (19-21).

Los procedimientos quirúrgicos para la evacuación uterina se consideran la estrategia más efectiva y segura para el tratamiento del aborto incompleto. El legrado con cureta metálica (también denominado dilatación y legrado) suele realizarse en quirófano, con anestesia general. En este método, se utiliza una cureta metálica para evacuar el contenido de útero. En la mayoría de los casos, el legrado con cureta se realiza sin dilatación del cuello uterino, ya que en el aborto incompleto el canal cervical generalmente se encuentra permeable (19-21).

La aspiración endouterina basa su mecanismo de acción en trasladar una presión negativa, generada por una fuente manual o eléctrica, dentro del útero, a través de unas cánulas que están conectadas a la fuente de presión negativa $(22,23)$. Este procedimiento permite retirar los tejidos endouterinos en su totalidad y evita raspar manualmente las paredes uterinas, con riesgo menor de daño en las mismas. La aspiración endouterina, tal como se conoce actualmente, puede ser realizada tanto en forma eléctrica como manual. La aspiración eléctrica requiere del uso de una bomba de vacío de tipo Berkeley que es operada con energía eléctrica. La AMEU utiliza un equipo portátil (jeringas con válvulas sencillas y dobles y cánulas de Karman) que no requiere del uso de electricidad, lo que permite su amplio uso en diferentes niveles del sistema de salud. Tiene las mismas ventajas de la aspiración eléctrica; sin embargo, su costo es mucho menor (24, 25).

El presente estudio se realizó con el objetivo de comparar la eficacia de la AMEU con el LUI como tratamiento del aborto incompleto o retenido en un grupo de pacientes que consultaron al Servicio de Sala de Partos de la Maternidad Concepción Palacios, en Caracas. 


\section{MÉTODOS}

Se realizó un estudio prospectivo, comparativo, transversal para evaluar dos técnicas: aspiración manual endouterina vs legrado uterino instrumental. Se incluyó una muestra probabilística de 192 pacientes con diagnóstico de aborto incompleto (expulsión parcial del producto de la concepción) o retenido ( $\sin$ expulsión del producto de la concepción). Se excluyeron aquellas con diagnóstico de aborto hemorrágico, séptico o quienes hubieran recibido tratamiento médico para el aborto. Todas las pacientes incluidas firmaron un formulario de consentimiento informado.

Las participantes fueron distribuidas en dos grupos al azar simple, a las del primer grupo se les realizó AMEU y a las del segundo grupo LUI, según las pautas regulares del servicio. Para la realización de la AMEU, las pacientes se colocaron en posición de litotomía, previa colocación de campos estériles y vaciamiento vesical, se introdujo el espéculo de metal tipo Graves y, posterior a realizar medidas de antisepsia, se aplicó anestesia local paracervical (lidocaína) en horas 4 y 8 con una jeringa de $5 \mathrm{cc}$, en cada punto se administró $2,5 \mathrm{cc}$ respectivamente, con aguja hipodérmica número 21 o 22; se colocó un tenáculo (pinza de Pozzi o Mousseaux) a nivel del labio cervical de la preferencia del operador y se procedió a ejercer tracción suave, se introdujo la cánula seleccionada según las dimensiones uterinas y la dilatación cervical, justo un poco más allá del orificio interno aplicando movimientos de rotación a medida que se ejercía una presión suave. Se conectó la cánula al aspirador y se liberó el vacío oprimiendo los botones para comenzar la aspiración; se practicó la evacuación del contenido del útero desplazando lenta y suavemente la cánula y el aspirador con movimientos de rotación de 180 grados en cada dirección y simultáneamente ejerciendo movimiento de adentro hacia afuera. Una vez finalizado el procedimiento se soltaron los botones y se retiró la cánula conectada del aspirador.

Vol. 81, $\mathrm{N}^{\circ} 2$, junio 2021
La paciente que se seleccionó para LUI, previa posición de litotomía, vaciamiento vesical y colocación de campos estériles, se le administró anestésicos endovenosos por parte del personal de anestesiología $\mathrm{y}$, una vez anestesiada la paciente, se efectuó antisepsia a nivel vaginal y cervical, previa a la colocación de valva vaginal, y aplicación de tenáculo (pinza de Pozzi o Mousseaux) a nivel de labio cervical de la preferencia del operador y se ejerció tracción suave; se procedió a introducir una cureta de Sims, seleccionada según la dilatación cervical y las dimensiones uterinas, y se realizó la extracción de los restos ovulares.

La evaluación de la cantidad del sangrado se hizo mediante valoración subjetiva según la impregnación de toalla sanitaria. La estimación del dolor se realizó mediante el empleo de la escala visual análoga (EVA) del dolor, durante el procedimiento, en las pacientes del grupo aspiración, y 10 minutos después del mismo, en ambos grupos; así como el requerimiento de analgésicos endovenosos. El dolor durante el procedimiento no fue evaluado en el LUI debido a que las pacientes de este grupo recibieron anestesia endovenosa. Se valoró de forma cuantitativa la eficacia del procedimiento mediante la evaluación ecográfica del diámetro del contenido de restos ovulares, previo al procedimiento y en un control dentro de las primeras 2 horas posterior al mismo. Se cuantificó la duración de cada procedimiento en minutos, desde que se colocó la paciente en posición de litotomía hasta finalizar la evacuación completa de cavidad uterina y retirar el material quirúrgico. Las complicaciones del procedimiento se apreciaron según la existencia o no de perforaciones uterinas, retención de restos ovulares e infección; y a su vez la tolerancia a los anestésicos según la presencia de episodios eméticos y la EVA. Se categorizaron las condiciones sociodemográficas de cada participante del estudio, en función de la edad, paridad, edad gestacional y cantidad de restos ovulares. Una vez obtenida toda la muestra se procedió a tabular los datos y se aplicó el tratamiento estadístico correspondiente. 
Se realizó una tabulación de los datos para indicar distribuciones de frecuencias, promedios, proporciones; con análisis inferenciales mediante $\chi^{2}$, valores $\mathrm{p}$ e intervalos de confianza de $95 \%$. Se consideró significativo cuando se obtuvo un valor de $\mathrm{p}<0,05$.

\section{RESULTADOS}

Las 192 pacientes que fueron incluidas, se distribuyeron al azar en dos grupos de 96 pacientes cada uno. Al evaluar los diagnósticos de ingreso se pudo observar que 150 pacientes correspondían a abortos incompletos y 42 a abortos retenidos, estos se distribuyeron así: en el grupo de LUI, 85 pacientes $(88,5 \%)$ tenían diagnóstico de aborto incompleto y $11(11,5 \%)$ eran abortos retenidos; para el grupo de AMEU, hubo 65 pacientes $(67,7 \%)$ con aborto incompleto y $31(32,2 \%)$ con aborto retenido.

La tabla 1 muestra las características generales de las pacientes en forma comparativa. La media de la edad fue 24,6 \pm ,6,68 años en el grupo de pacientes a quienes

Tabla 1. Comparación de las características generales de las pacientes

\begin{tabular}{|c|c|c|c|}
\hline Variables & $\begin{array}{c}\text { Legrado } \\
\text { uterino } \\
\text { instrumental } \\
\mathrm{n}=96\end{array}$ & $\begin{array}{c}\text { Aspiración } \\
\text { manual } \\
\text { endouterina } \\
\mathrm{n}=96\end{array}$ & $\mathrm{p}$ \\
\hline Edad (años)* & $24,6 \pm 6,68$ & $25,4 \pm 7,78$ & 0,5377 \\
\hline Paridad** & & & 0,5423 \\
\hline Primigesta & $23(24 \%)$ & $31(32,3 \%)$ & \\
\hline II a IV gestas & $70(72,9 \%)$ & $58(60,4 \%)$ & \\
\hline V o más gestas & $3(3,1 \%)$ & $7(7,3 \%)$ & \\
\hline $\begin{array}{l}\text { Edad gestacional } \\
(\text { semanas)* }\end{array}$ & $9,1 \pm 2,6$ & $9,2 \pm 2,5$ & 0,5646 \\
\hline $\begin{array}{l}\text { Grosor endometrial } \\
(\mathrm{mm})^{*}\end{array}$ & $22,3 \pm 4,43$ & $23,9 \pm 4,39$ & 0,5001 \\
\hline
\end{tabular}

se les practicó LUI y 25,4 \pm 7,78 años en el grupo de aquellas a quienes que se le realizó AMEU. En ambos grupos se observó que la mayoría de las pacientes eran multíparas, $72,9 \%$ en el grupo de LUI y $60,4 \%$ en el grupo de AMEU. La edad gestacional promedio fue 9,1 y 9,2 semanas, respectivamente. El promedio del grosor endometrial evaluado en cada grupo al momento de su ingreso fue $22,3 \pm 4,43 \mathrm{~mm}$ para el grupo LUI y 23,9 $\pm 4,39 \mathrm{~mm}$ para el grupo AMEU. Las diferencias entre los grupos no son significativas, por lo tanto, son comparables.

En la tabla 2 se puede observar que la cantidad de restos ovulares constatados por ecografía transvaginal posterior al LUI o la AMEU no superó los $5 \mathrm{~mm}$ de longitud ( $\mathrm{p}=0,2145)$.

Al aplicar la EVA en el grupo AMEU durante el procedimiento, se observó que 93 pacientes $(96,9 \%)$ manifestaron sentir dolor de leve intensidad (1-3 puntos) y solo 3 pacientes $(3,1 \%)$ presentaron dolor moderado (4-6 puntos). Al medir la intensidad del dolor después de la realización de los procedimientos,

Tabla 2. Distribución comparativa de pacientes según la medida de los restos ovulares después de cada procedimiento

\begin{tabular}{lcc}
\hline $\begin{array}{l}\text { Restos } \\
\text { postratamiento } \\
(\mathrm{mm})\end{array}$ & $\begin{array}{c}\text { Legrado } \\
\text { uterino } \\
\text { instrumental } \\
\mathrm{n}(\%)\end{array}$ & $\begin{array}{c}\text { Aspiración } \\
\text { manual } \\
\text { endouterina } \\
\mathrm{n}(\%)\end{array}$ \\
\hline 1 & 0 & $1(1,0)$ \\
2 & $6(6,3)$ & $9(9,4)$ \\
3 & $33(34,4)$ & $37(38,5)$ \\
4 & $40(41,7)$ & $34(35,4)$ \\
5 & $17(17,7)$ & $15(15,6)$ \\
\hline
\end{tabular}

$\mathrm{p}=0,2145$ 
Tabla 3. Distribución comparativa según el dolor después del procedimiento

\begin{tabular}{lcc}
\hline $\begin{array}{l}\text { Intensidad del dolor } \\
\text { (puntos) }\end{array}$ & $\begin{array}{c}\text { Legrado } \\
\text { uterino } \\
\text { instrumental } \\
\mathrm{n}(\%)\end{array}$ & $\begin{array}{c}\text { Aspiración } \\
\text { manual } \\
\text { endouterina } \\
\mathrm{n}(\%)\end{array}$ \\
\hline 0 & $29(30,2)$ & $24(25)$ \\
1 & $47(48,9)$ & $50(52,1)$ \\
2 & $19(19,8)$ & $20(20,8)$ \\
3 & $1(1,1)$ & $2(2,1)$ \\
$\mathrm{p}=0,9174$ & &
\end{tabular}

en la tabla 3 se puede evidenciar que el dolor fue descrito como ausente por 29 mujeres del grupo LUI y 24 del grupo AMEU. Sesenta y siete pacientes sometidas a LUI y 72 del grupo AMEU describieron el dolor como leve (puntuación entre 1 y 3 en la EVA) $(\mathrm{p}=0,9174)$.

En el grupo de AMEU no se presentaron efectos colaterales relacionados con la anestesia, mientras que entre las pacientes a quienes se les practicó LUI se observó náuseas en 2 casos $(2 \%)$ y mareos en 1 $(1 \%)$. No se presentaron complicaciones inherentes al procedimiento (retención de restos, perforaciones ni infección) en ninguno de los dos grupos.

El tiempo mínimo de duración del LUI fue de 5,3 minutos y el máximo de 12 minutos, con una media de 8,45 $\pm 1,42$ minutos; para la AMEU los extremos fueron 6 minutos y 12 minutos y la media fue 8,50 \pm 1,32 minutos $(\mathrm{p}=0,8138)$.

En relación a la cantidad de sangrado después de realizarse los procedimientos, se puede evidenciar en la tabla 4 que en ambos grupos fue predominantemente entre 5 y $9 \mathrm{ml}: 50$ pacientes (52\%) en el grupo legrado y 62 pacientes $(64,6 \%)$ en el grupo AMEU. El valor máximo fue de $15 \mathrm{ml}$ para ambos grupos.
Tabla 4. Distribución comparativa de pacientes según el volumen del sangrado postratamiento

\begin{tabular}{lcc}
\hline $\begin{array}{l}\text { Sangrado } \\
\text { postratamiento } \\
(\mathrm{ml})\end{array}$ & $\begin{array}{c}\text { Legrado } \\
\text { uterino } \\
\text { instrumental } \\
\mathrm{n}(\%)\end{array}$ & $\begin{array}{c}\text { Aspiración } \\
\text { manual } \\
\text { endouterina } \\
\mathrm{n}(\%)\end{array}$ \\
\hline 0 & $3(3,1)$ & 0 \\
$1-4$ & $22(23)$ & $17(17,7)$ \\
$5-9$ & $50(52)$ & $62(64,6)$ \\
$10-14$ & $17(17,7)$ & $13(13,5)$ \\
15 & $4(4,2)$ & $4(4,2)$ \\
\hline
\end{tabular}

$\mathrm{p}=0,4082$

\section{DISCUSIÓN}

A nivel mundial $13 \%$ de las muertes maternas se atribuyen a complicaciones del aborto, de los cuales $98 \%$ se producen en países en vías de desarrollo, y es en América del Sur donde se sitúa la tasa más alta (2-5). El aborto representa una causa importante de morbimortalidad materna a nivel mundial, lo que obliga a los sistemas de salud a aplicar medidas eficaces y seguras para el tratamiento del mismo; en virtud de lo cual la OMS recomienda la AMEU como primera opción terapéutica en el tratamiento quirúrgico del aborto incompleto, con base en las ventajas que ofrece en comparación al legrado uterino instrumental que han sido reportadas en diversos estudios comparativos (2, 6-8). El presente estudio se realizó con el objetivo de comparar la eficacia de la AMEU con el LUI como tratamiento del aborto incompleto o retenido.

Dentro de las ventajas que se describen de la aspiración manual endouterina está el alcanzar una efectiva evacuación de la cavidad, en tanto que la retención de restos ovulares resulta una complicación poco frecuente, tal como lo describen diversos autores en varios estudios coincidiendo con los resultados obtenidos en este estudio; sin embargo Farooq y cols. (13) describieron que la retención de restos ovulares 
es la única complicación presentada en el grupo de AMEU.

Con relación a la tolerancia a la técnica quirúrgica, vale la pena recalcar que todas las pacientes a quienes se les aplicó AMEU toleraron el procedimiento y ninguna de ellas tuvo algún efecto adverso relacionado con la aplicación de anestesia paracervical o inherente al tratamiento. Por lo que, en este sentido, la AMEU tiene una doble ventaja, no necesita del personal de anestesiología para llevarse a cabo y la aplicación de anestesia local no ocasiona grandes complicaciones. También hay que sumarle la importancia que merece la disminución de los gastos hospitalarios como consecuencia de lo antes expuesto y considerar, además, el ahorro de la estancia hospitalaria por el menor tiempo de recuperación que ameritan las pacientes posterior al procedimiento, si el mismo es realizado con la aplicación local de fármacos anestésicos.

Si bien en la mayoría de los estudios que anteceden a este se ha podido constatar que la duración del procedimiento es menor en la AMEU, es notorio que casi la totalidad de ellos solo han evaluado la evacuación uterina en gestaciones menores a 13 semanas $(6,9$ - 13, 17). Contrariamente, este estudio reporta un tiempo similar en ambos grupos, 8 minutos en promedio, sin diferencia estadísticamente significativa $\mathrm{p}=0,8138$, en contraste al tiempo reportado por Forna y cols. (9) quienes señalaron que la duración del procedimiento fue más corta para la AMEU que para LUI (-1,2 minutos de diferencia de promedios ponderados; IC del $95 \%$ : $-1,5$ a -0.87 minutos); es importante mencionar el hecho de que los procedimientos en ambos grupos fueron realizados por residentes de posgrado de distintos niveles lo que pudo afectar la duración, porque aquellos de menor nivel son considerados con menor destreza al momento de realizar los mismos.
Se concluye que tanto la AMEU como el LUI son eficaces como tratamiento quirúrgico del aborto incompleto o retenido, teniendo la capacidad de evacuar la cavidad uterina satisfactoriamente y sin complicaciones relacionadas al procedimiento quirúrgico o muy escasas en relación al acto anestésico, siendo estas últimas nulas en el caso de emplearse la AMEU.

\section{REFERENCIAS}

1. Cabero RA, Martínez P. Aborto de repetición: causa, diagnóstico y tratamiento. En: Cabero RL, Saldívar RD, Cabrillo RE, editores. Obstetricia y Medicina MaternoFetal. $1^{\text {a }}$ ed. Madrid: panamericana; 2007. p 521-530.

2. World Health Organization [Internet]. Ginebra: Safe Abortion: Technical and Policy Guidance for Health Systems. Geneva: World Health Organization; 2012 [consultado octubre de 2016]. Disponible en: https:// www.who.int/reproductivehealth/publications/unsafe abortion/9789241548434/en/

3. Ahman E, Shah IH. New estimates and trends regarding unsafe abortion mortality. Int J Gynaecol Obstet. 2011; 115(2):121-126. doi: 10.1016/j.ijgo.2011.05.027.

4. Távara L. Contribución de las Sociedades de Obstetricia y Ginecología de América del Sur para la prevención del aborto inseguro en la región. Int J Gynaecol Obstet. 2014; 126(2014): S7-S9. doi: http://dx.doi. org/10.1016/j.ijgo.2014.03.011

5. Barroso C, Langer A. Salud y Derechos Sexuales y Reproductivos en América latina y el Caribe: desafíos y oportunidades [Internet]. Grupo de trabajo regional para la reducción de la mortalidad materna; 2009 [consultado octubre de 2016]. Disponible en: https:// clacaidigital.info/bitstream/handle/123456789/530/ G T R r ed u c c i on mortalidadmaterna . pdf? sequence $=5$ \&isAllowed $=\mathrm{y}$

6. Tunçalp O, Gülmezoglu AM, Souza JP. Surgical procedures for evacuating incomplete miscarriage. Cochrane Database Syst Rev. 2010; 2010(9):CD001993. doi: 10.1002/14651858.CD001993.pub2.

7. Lean TH, Vengadasalam D, Pachauri S, Miller ER. A comparison of $\mathrm{D} \& \mathrm{C}$ and vacuum aspiration for performing first trimester abortion. Int $\mathrm{J}$ Gynaecol Obstet. 1976; 14(6):481-486. doi: 10.1002/j.18793479.1976.tb00089.x. PMID: 20343. 


\section{ASPIRACIÓN MANUAL ENDOUTERINA EN COMPARACIÓN CON EL LEGRADO UTERINO INSTRUMENTAL EN EL TRATAMIENTO DEL ABORTO INCOMPLETO O RETENIDO}

8. Schweppe KW, Wagner H, Beller FK. [Abortion by means of suction curettage compared to the conventional metal curettage]. Med Welt [Internet]. 1980 [consultado octubre de 2016]; 31(13):479-83. German. PMID: 7382809. Disponible en: https:// pubmed.ncbi.nlm.nih.gov/7382809/

9. Forna F, Gülmezoglu AM. Surgical procedures to evacuate incomplete abortion. Cochrane Database Syst Rev. 2001; (1):CD001993. doi: 10.1002/14651858. CD001993. Update in: Cochrane Database Syst Rev. 2010; (9):CD001993.

10. Pereira PP, Oliveira AL, Cabar FR, Armelin AR, Maganha CA, Zugaib M. [Comparative study of manual vacuum aspiration and uterine curettage for treatment of abortion]. Rev Assoc Med Bras. 2006; 52(5):304-307. Portuguese. doi: 10.1590/s0104-42302006000500015.

11. Cisse CT, Faye KG, Moreau JC. Avortements du premier trimestre au chu de Dakar: interêt de l'aspiration manuelle intra-uterine [First-trimester abortion at University Hospital Center in Dakar, Senegal: utility of manual vacuum aspiration]. Med Trop (Mars) [Internet]. 2007 [consultado octubre de 2016]; 67(2):163-6. French. Disponible en: https:// pubmed.ncbi.nlm.nih.gov/17691436/

12. Kulier R, Fekih A, Hofmeyr GJ, Campana A. Surgical methods for first trimester termination of pregnancy. Cochrane Database Syst Rev. 2001;(4):CD002900. doi: 10.1002/14651858.CD002900.

13. Farooq F, Javed L, MumtazA, Naveed N. Comparison of manual vacuum aspiration, and dilatation and curettage in the treatment of early pregnancy failure. J Ayub Med Coll Abbottabad [Internet]. 2011 [consultado octubre de 2016]; 23(3):28-31. Disponible en: https://pubmed. ncbi.nlm.nih.gov/23272429/

14. Saciloto MP, Konopka CK, Velho MT, Jobim FC, Resener EV, Muradás RR, et al. [Manual vacuum aspiration uterine treatment of incomplete abortion to 12 gestational weeks: an alternative to curettage]. Rev Bras Ginecol Obstet [Internet]. 2011 [consultado octubre de 2016]; 33(10):292-296. Portuguese. Disponible en: https://pubmed.ncbi.nlm.nih.gov/22231162/

15. Choobun T, Khanuengkitkong S, Pinjaroen S. A comparative study of cost of care and duration of management for first-trimester abortion with manual vacuum aspiration (MVA) and sharp curettage. Arch Gynecol Obstet. 2012; 286(5):1161-1164. doi: 10.1007/ s00404-012-2405-4.
16. Yonke N, Leeman LM. First-trimester surgical abortion technique. Obstet Gynecol Clin North Am. 2013; 40(4):647-670. doi: 10.1016/j.ogc.2013.08.006.

17. Kinariwala M, Quinley KE, Datner EM, Schreiber CA. Manual vacuum aspiration in the emergency department for management of early pregnancy failure. Am J Emerg Med. 2013; 31(1):244-247. doi: 10.1016/j. ajem.2012.07.014.

18. Gilman Barber AR, Rhone SA, Fluker MR. Curettage and Asherman's syndrome-lessons to (re-) learn? J Obstet Gynaecol Can. 2014; 36(11):997-1001. doi: 10.1016/S1701-2163(15)30413-8.

19. Organización Mundial de la Salud [Internet]. Montevideo: Manual de práctica clínica para un Aborto Seguro; 2014 [consultado octubre 2016]. Disponible en: https:/www.who.int/reproductivehealth/publications/ unsafe_abortion/clinical-practice-safe-abortion/es/

20. Alarcón M. El legrado uterino, indicaciones, beneficios y riesgos. Guías de manejo. Médicas UIS [Internet]. 2007 [consultado octubre de 2016]; 20(2): 151-156. Disponible en: https://revistas.uis.edu.co/index.php/ revistamedicasuis/article/view/1837/2215

21. Chumbe O. Manejo del aborto. Rev Peru Ginecol Obstet [Internet]. 2008 [consultado octubre de 2016]; 54: 244-248. Disponible en: https://www.redalyc.org/ articulo.oa? $\mathrm{id}=323428190004$

22. Greenslade FC, Leonard AH, Benson J, Winkler J, Henderson VL. Manual vacuum aspiration: a summary of clinical and programmatic experience worldwide. Carolina del Norte: Ipas; 1993.

23. Blumenthal PD, Remsburg RE. A time and cost analysis of the management of incomplete abortion with manual vacuum aspiration. Int J Gynaecol Obstet. 1994; 45(3):261-267. doi: 10.1016/0020-7292(94)90252-6.

24. Solter C, Miller S Gutiérrez M. A comprehensive training course: manual vaccum aspiration (MVA) for treatment of incomplete abortion. Primera edición. Boston: Pathfinder International; 2000.

25. Gutiérrez M, Guevara E, Flores L, Acevedo E, Luna A. Módulo de Capacitación Atención Integral del Aborto Incompleto no Complicado. Segunda Edición. Lima: Pathfinder International; 2011.

Recibido 28 de julio de 2020 Aprobado 10 de octubre de 2020 